\title{
Survey on the Mechanisms of Organic Solar Cell
}

\author{
Yiyang $\mathrm{Li}^{1,}$ a \\ ${ }^{1}$ Faculty of Engineering and Information Technologies, The University of Sydney, Sydney, NSW, \\ Australia, 2006. \\ ayili3929@uni.sydney.edu.au
}

Keywords: organic solar cell; mechanisms; photovoltaic effect.

\begin{abstract}
With the rapid development of conductive polymers, the study of low cost solar cells becomes possible. Because of huge potential application value of conducting polymers, organic solar cell research is the hotspot of applied research in this field. This paper studied the mechanism of the organic polymer solar cells and the photoelectric conversion process of organic solar cells, and analyzed the loss mechanism in the process of photoelectric conversion. Through the analysis of the mechanism of organic polymer solar cells, this paper provided the basis of theory research for the preparation of high conversion efficiency of composite organic polymer solar cells.
\end{abstract}

\section{Introduction}

In recent years, the rapid development of conductive polymers makes it possible to research and develop the low-cost organic solar cells. Because of huge potential application value of conducting polymers, polymer solar cells research is the hotspot of applied research in this field. During the gradually mature of theory study, a new organic conductive materials appeared, the physical and chemical properties of new materials are also gradually known by people ${ }^{[1,2]}$.Conjugating conductive polymer materials due to the workability and flexibility of both polymer and inorganic semiconductor features or metal electrical conductivity, thus has great potential commercial value. Due to it has the workability and flexibility of polymer and the features of inorganic semiconductor or metal electrical conductivity, thus conjugating conductive polymer materials has great potential commercial value. Because of raw material price is low, the production process is simple and can be widespread preparation, if there is a further breakthrough on the performance of polymer solar cells, it will increase the efficiency of energy conversion close to the level of commercialization of inorganic materials of solar cells, and it is likely to be large-scale used widely, and its market prospects will be very great.

In this paper the mechanism of organic polymer solar cells were reviewed, including the conductivity of organic polymer materials, the generation principle of charge carrier of organic polymer solar cells, as well as the photoelectric conversion process of organic solar cells, and this paper also analyzed the loss mechanism in the process of photoelectric conversion.

\section{Conductive Performance of Organic/Polymer}

Unlike metal conductor, organic materials including polymers are exist in the form of the molecule. When organic compounds with conjugate structure, $\pi$ electronic system are increased, electron delocalization are also increased, and movable scope are expanded. When the conjugate structure is large enough, the compound can provide free electrons. The bigger conjugate system is, the more delocalization. Therefore the necessary condition of organic polymer becomes conductor is that there is a conjugate structure can make the internal some electrons or holes possess the delocalized across bond and move power ${ }^{[3]}$.

The conductive ability of conductive polymers without doping is still within the scope of semiconductor material, because each pi bond molecular orbital of the pure or not doped conductive polymer molecules still exist energy level difference. Under the action of electric field force, electrons migrate within the polymer must across the energy level difference. The existence of the 
energy level difference, causing two valence electrons cannot be totally free in the conjugate polymer across bond move. Thus its conductive ability is affected, the conductivity is not high, according to its conductive capability it should belong to the category of semiconductor, and the conductive ability of electronic conductive polymer without doping is equal to the typical inorganic semiconductor materials such as germanium and silicon, and also has a certain gap to the metal conductor in conductive ability. The relative migration is the basis of the conduction electrons. Therefore, if the electron want to move freely in the conjugate electronic system, the first thing is to overcome energy level difference between the full filled band and empty band. The reason is that full filled band and empty band have interval with each other in the molecular structure, and the energy level difference determines the size of the conjugate polymer conductive ability. It is because the energy level difference determines we get is not a good conductor but semiconductor.

Due to the electrons in the valence band is localized, conjugate polymer has no contribution to conductivity. Whether it has electrical conductivity, mainly depends on the forbidden band width. In addition, the barrier between the molecules is very high, chain length of the chain is not equal, three-dimensional obstacles in side chain is to lower conductivity, therefore, conjugate polymer general electrical conductivity is very low.

As active materials of organic photovoltaic devices must have the function of conjugate system exist in the molecular chain and finished light absorption with a part of $\pi$ delocalization and $\pi^{\wedge *}$ track and charge transfer process.

\section{Carrier Generate Principle of Organic /Polymer Solar Cells}

According to the electron affinity, organic polymer semiconductor materials can be divided into electron donor material and electron acceptor material. Electron donor materials with low ionization potentials, can receive hole from the valence band of other contact with organic material, and thus become empty hole acceptor or electron donor. Electron acceptor material has larger electron affinity, can receive electronic from the conduction band of other materials, therefore as electron acceptor. In organic polymer semiconductor materials, the Highest Occupied Molecular Orbital was used to express the orbit that the highest electron energy level was possessed, and it is called the highest occupation orbit; and use the Lower Unoccupied Molecular Orbital was used to express the orbit that the lowest electron energy level was not possessed, and it is called the minimum not occupation orbit. HOMO and LUMO are collectively referred to the front rail, electron in the front line electronic was called the front electron. Frontier orbital theory think that there is electron which is similar to the individual atoms "valence electrons" exist in molecules, molecular electronic valence is the front electrons, so in the process of chemical reaction between molecular, the role of the molecular orbital is the front rail, the front line electron plays a key role. This is because HOMO molecules has a loose constraints of its electron relatively, and it has the nature of the electron donor, but LUMO has a stronger affinity of its electron, and it is the nature of the electron acceptor. This two track are most likely to interact with each other, and plays an extremely important role in the process of chemical reaction ${ }^{[4]}$.

When organic molecules absorb photons and be excited, it is quite different from inorganic semiconductor. The binding energy between electron-empty hole which are produced by silicon semiconductor photons is small, and it can effectively thermal ionization at room temperature, thus forming the free carrier. Optical excitation of organic molecules, and produced empty hole-electron pair which is electrostatic interactions together, known as Exciton. Binding energy of exciton is bigger, its absolute value is the hot research subjects of the polymer semiconductor, and generally it is about $0.4 \mathrm{eV}$. Because the polymer semiconductor has strong exciton binding energy, the expression of exciton is electrically neutral, and the migration of current without any contribution.

At the same time the existence time of exciton is limited, usually it is under the millisecond level, electrons and holes will recombination without thorough separation, and release its energy. The separation efficiency of exciton in organic semiconductor has the key effect on photoelectric conversion efficiency of the battery. 
As shown in figure 1, in order to generate the photocurrent, the dissociation of exciton is appeared in the polymer, or at the interface between metal electrode/polymer molecular. Only be dissociated into free charge carriers (electrons and empty holes), exciton can produce the photocurrent. Exciton dissociation has a variety of mechanisms, including thermal ionization of exciton or freedom ionization, collisions ionization between the exciton and exciton, photoionization, and exciton interact with impurities or defect centers and ionization, etc. The mobility of free carrier which is produced by ionization is low, and it is very easy to compound in pairs and losses. Only the exciton spread to the electrode/donor/receptor of interface, and the interface built-in dissociation to contribute to the photocurrent.

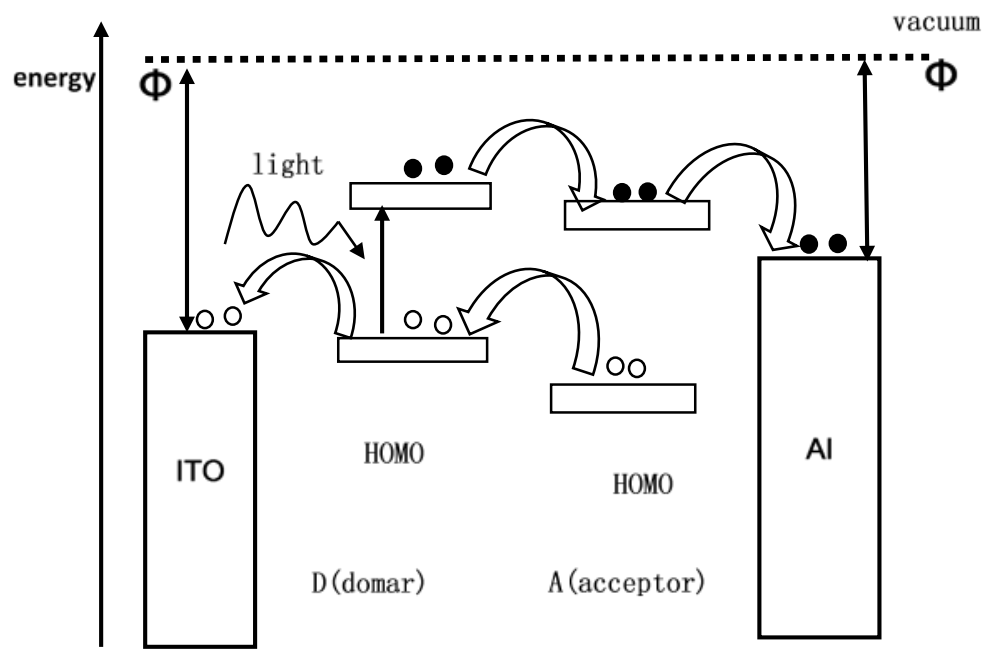

Fig. 1 Generation Process of Carrier

\section{Generate Principle of Organic Carrier/polymer Solar Cells}

Analysis of the conversion process organic polymer solar cells photoelectric has very important significance on further understanding of the physical mechanism of the organic polymer solar cells, and the improvement of the photoelectric conversion efficiency of the device.

\subsection{The photon absorption.}

When the frequency range of incident light to a certain thickness plane media, within the medium will produce light waves because of reflection of both the before and after interface, these light and incident light was overlay each other. If the thickness of the medium is not larger than the wavelength of the incident light, medium can produce significant light wave interference effect, and formed photoelectric field because the superimposition of the incident wave and reflected wave. For organic polymer solar cells, the film thickness is usually $30 \sim 200 \mathrm{~nm}$, and it is far smaller than the wavelength of incident light. Therefore the influence of optical interference effect on the optical field distribution in the device must be considered.

In most of the organic polymer devices, only a small fraction of incident light is absorbed by the device. The main reason is that the band gap of organic polymer materials is too high, most of the band gap is higher than $2.0 \mathrm{Ev}(600 \mathrm{~nm})$, and only absorbs about $30 \%$ of the solar radiation. Organic active layer is too thin and also limits the absorption of light. Because of the diffusion distance limitation of exciton, so the thickness of the thin film generally is $30 \sim 200 \mathrm{~nm}$, and it affected the absorption of light. The absorption rate of organic polymer materials with thickness about $100 \mathrm{~nm}$ is $60 \% \sim 90 \%$. Reflection loss is the reason that cause of photon absorption is less.

Fortunately, the absorption of the organic polymer light wave range is very narrow, but the light absorption coefficient and inorganic silicon is much higher (about $105 \mathrm{~cm}-1$ ), the thickness about $100 \mathrm{~nm}$ of organic polymer materials in the optical density can get higher. Polymer has big $\pi$ - 
conjugate system, and there is wide $\pi$ and $\pi$ e band, but the band gap can be adjust by mixed or chemical modification, and absorb sunlight effectively.

\subsection{The generation and diffusion of exciton.}

Electron hole which is produced by organic molecules stimulated by light is exists in the form of exciton. The existence time of exciton is limited, and it is usually under the orders of milliseconds magnitude, so the exciton has certain spread distance. In polymers and pigments the diffusion distance is about $10 \mathrm{~nm}$. The diffusion distance of exciton is about several hundred nanometers in some pigments. If the exciton failed to spread to the interfaces and dissociated, electrons and holes will compound without complete separation, and releases its absorbed energy.

\subsection{Charge separation.}

Exciton arrive at the interface in diffusion length. The interface including organic semiconductor metal interface, polymer metal interface, D/A interface. Under the influence of built-in electric field of the interface, exciton dissociated into free carrier electrons and holes.

\subsection{Charge transport.}

Free carrier get to an electrode through different transport channels. Electronic transfer to the cathode in receptor material and hole transfer to ITO anode in receptor material. Electrons and holes are prone to compound in the process of transport to the electrode, in particular, some material can serve as electronic transmission material at the same time hole-transporting materials. The interaction of carriers and atoms or ions will affect its migration in the process of transmission. Compared with silicon semiconductor, the interaction between organic molecules is much weaker, LUMO and HOMO cannot be formed continuous conduction band and valence band through the combination in the whole bulk phase. The transport of carrier in organic semiconductors need to implement through the "jump" mechanism of the charge between different molecular, the macro performance is that its carrier mobility was much lower than inorganic semiconductor.

\subsection{Charge collection.}

Free carrier transport to the electrode, and it is collected near the electrode. work function of electrode material is very important in the organic devices, because it can according to the HOMO and LUMO Fermi level of the organic polymer semiconductor materials to determine whether the contact of electrode with electrons and holes is ohmic contact or not. And the big difference value of the work function in the two materials can increase the open circuit voltage of the device. Collect electronic electrode material requirements with low work function, such as $\mathrm{Al}, \mathrm{Ca}, \mathrm{In}, \mathrm{Ag}$, and so on, this collecting hole of electrode materials demand higher work function, ITO, Au, etc.

The absorption in organic solar cells activate region must satisfy that the free carrier photons must enough after absorbed, and suggest there is internal electric field produced by the carrier in transit must arrive at the external circuit is low loss. There are a lot of losses in a photoelectric conversion process, however, it makes the conversion efficiency of organic polymer photovoltaic cells photoelectric low. Figure 2 give a sign for the photoelectric conversion process and the loss mechanism. 


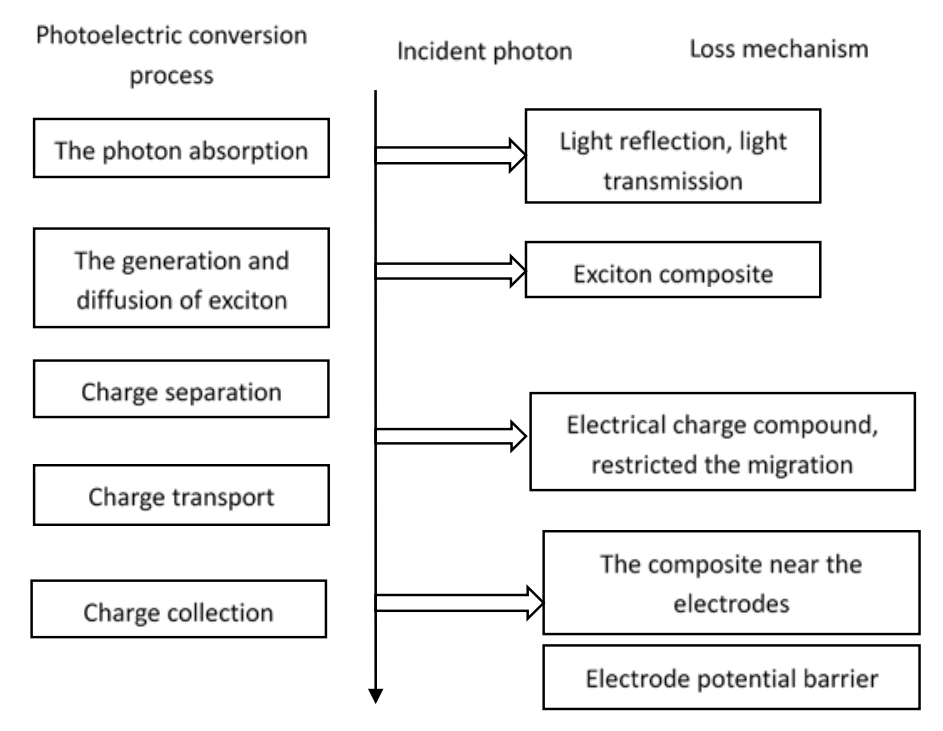

Fig. 2 The photoelectric conversion process and loss mechanism

\section{Conclusions}

This paper studied the mechanism of the organic polymer solar cells and the photoelectric conversion process of organic solar cells, and also analyzed the loss mechanism in the process of photoelectric conversion. Through the analysis of the mechanism of organic polymer solar cells, this paper provided the basis of theory research for the preparation of high conversion efficiency of composite organic polymer solar cells.

\section{References}

[1]. Katsumi Yashino. Novel electrical and optical properties of liquid conducting polymers and oligomers. IEEE Transactions On Dielectrics and Electrical Insulation, I 994, 1(3): 353 364

[2]. I. D.Norris, L. A. P.Kane, G. G.Wallace. Thermochromism in Optically Active Polyaniline Salts. Macromolecules, 1998, 31(19): 6529 6533

[3]. P M Grant, T Tani, W D Krounbi, et al. Properties of metal/polyac-etylene schottky barriers. Appl. Phys. Lett., 1981, 52(2): 869 872

[4]. You, J., Dou, L., Yoshimura, K., Kato, T., Ohya, K., Moriarty, T., ... \& Yang, Y. (2013). A polymer tandem solar cell with $10.6 \%$ power conversion efficiency.Nature communications, 4 , 1446. 